\title{
Evaluation of Pupil Diameter and Midline Shift in Patients Undergoing Decompressive Craniectomy
}

\author{
๑ Aykut Akpinar, ๑ Uzay Erdogan*, • Gurkan Berikol* * \\ University of Health Sciences Turkey, Haseki Training and Research Hospital, Clinic of Neurosurgery, Istanbul, Turkey \\ *University of Health Sciences Turkey, Ministry of Istanbul Bakirkoy Prof. Dr. Mazhar Osman Mental Health and Neurological Diseases \\ Training and Research Hospital, Clinic of Neurosurgery, Istanbul, Turkey \\ **Taksim Training and Research Hospital, Clinic of Neurosurgery, Istanbul, Turkey
}

\section{Abstract}

\begin{abstract}
Aim: Intracranial pressure may increase due to reasons such as head trauma, intracranial hematoma, cerebral infarction, and cerebral edema. Decompressive craniectomy (DC) may be preferred in the treatment. Our study aims to examine the time of DC and the effect of pupil diameter and midline shift on mortality.
\end{abstract}

Methods: Data from the hospital automation system and patient file records were retrospectively reviewed between 2017 and 2019 , and 57 patients who underwent DC for raised intracranial pressure. Fifty-seven patients who underwent DC, whose neurological examination and pupil diameter were recorded after increased intracranial pressure, were evaluated.

Results: The mean age of 57 patients was $50.38 \pm 19.91$ years. The patients' mean preoperative Glasgow coma score (GCS) was $8.85 \pm 3.6$ and the mean GCS at discharge was $8.96 \pm 6.0$. The mean cranial midline shifts of the patients were $7.88 \pm 5.35 .49 .1 \%$ of the patients who underwent DC survived.

Conclusion: Mortality rate is lower in patients who underwent DC compared to patients with isochoric pupil, anisocoric, and mid dilated patients. The timing of DC is important in terms of its effect on mortality.

Keywords: Decompressive craniectomy, intracerebral hemorrhage, intracranial hypertension, trauma

\section{Introduction}

Intracerebral hemorrhage can cause an increase in intracranial pressure (ICP) as a result of traumatic brain injury, cerebral infarction, and cerebral edema. Early intubation, hyperventilation, hyperosmolar therapy, barbiturate coma, and external ventricular drainage system are recommended for reducing intracranial pressure. Decompressive craniotomy (DC) can be planned in cases that do not respond to treatment $(1,2)$. DC is a surgical procedure performed by removing a large portion of the skull and opening the dura mater. With DC, a sufficiently large bone flap should be removed to reduce intracranial pressure and increase compliance. It has been shown that functional outcomes are better with adequately large DC sizes (3). Except for three; subtemporal, bifrontal, frontotemporoparietal types, unilateral and bilateral DC has been defined. While it is frequently performed in the frontotemporoparietal area in cerebral infarction cases, bifrontal decompressive surgery is frequently performed in cases of frontal contusion cerebri and edema $(4,5)$.

Decompressive hemicraniectomy and bifrontal craniectomy are the most commonly used surgical procedures. DC can be applied in early and late periods. However, the application time of DC is still controversial (6-8). In addition, there are few articles in the literature reporting the effect of pupil diameter, midline shift status on DC application time, and mortality.

Our study aimed to evaluate the effect of DC on the time and mortality of pathology differences that increase intracranial pressure by examining GCS, midline shift, pupil diameter. 


\section{Methods}

\section{Study Design}

Ethical approval was obtained from the Ethics committee of University of Health Sciences Turkey, Haseki Training and Research Hospital (protocol number: 149, date: 17.6.2019). Data from the hospital automation system and patient file records were retrospectively reviewed between 2017-2019 and 57 patients who underwent DC for raised intracranial pressure (cerebrovascular infarction, post-traumatic subarachnoid hemorrhage, epidural hemorrhage, subdural hemorrhage, intracranial hemorrhage), abscess, postoperative tumor and aneurysm) were included in the study. Patients who did not undergo DC and whose file information and examinations were missing were excluded from the study. Age, gender, hypertension, diabetes mellitus, anticoagulant usage status, neurological examination and pupil diameters, Glasgow coma scale (GCS), pupillary light reflex, cranial midline shift, and survival were recorded.

DC was performed unilaterally in all patients. Frontotemporoparietal DC was performed in 48 patients, subtemporal in 3 patients and bifrontal DC in 6 patients.

\section{Surgical Technique}

Surgery was performed under general anesthesia. The surgical field was closed sterile. The periosteum was resected with a skin flap from the incision line. The burr hole was opened with a hand motor. Bone tissue was removed by joining around the burr holes with the hand motor cutting edge and craniectomy was performed. The dura was opened with a scalpel. The dura was suspended from the sides to the bone tissue. The hematoma was evacuated in patients with hematoma. Bleeding control was performed in a controlled manner at these stages. A drain was placed in the subdural space. The layers were closed in accordance with the anatomical structure.

\section{Statistical Analysis}

SPSS ${ }$ for Windows v20.0 (SPSS Inc., Chicago, IL, USA was used for statistical analysis. The frequency, arithmetic mean and the standard deviation of the variables were shown in descriptive data. The distributions were calculated using the Kolmogorov-Smirnov normality test and the differences among the independent groups were calculated by independent samples (Student's t-test) for normal distribution.

\section{Results}

The mean age was $50.38 \pm 19.91$ years old. Twentythree (40.4\%) patients had hypertension, 7 (12.3\%) patients had diabetes mellitus. The anticoagulant use rate was $28.1 \%$. The mean GCS before decompressive surgery was $8.85 \pm 3.6$ and $8.96 \pm 6.00$. Twenty-nine $(50.9 \%)$ after decompressive surgery (Table 1). Twenty-eight (49.1\%) of the patients were survived.

Nine $(15.8 \%)$ of the operated patients had cerebral infarction. Twenty-four patients (42.1\%) had a subarachnoid hemorrhage, epidural hemorrhage, and subdural hemorrhage due to trauma. There were 12 $(21.1 \%)$ bleeding complications after tumor surgery, 2 (3.5\%) bleeding after aneurysm surgery, 9 (15.8\%) primary intracranial hemorrhage, and 1 (1.8\%) cranial abscess (Graphic 1).

Decompressive surgery direction of the patients; right side $40.4 \%$, left side $50.8 \%$, frontal or occipital $7 \%$, and bilateral $1.8 \%$. In $70 \%$ of the patients, galeal duraplasty was performed after durotomy during surgery. In the pupil examination of the patients; $12.3 \%(n=7)$ pupils were fix or mid-dilated, $38.6 \%(n=22)$ had anisocoria, $49.1 \%(n=$ 28) were isochoric. The distribution of ages was $33.3 \%$ $(n=19)$ under 40, 29.8\% ( $n=17)$ between 40 and 60, $36.8 \%(n=21)$ over 60 years old respectively. When the

\begin{tabular}{|c|c|c|c|c|}
\hline & & Mean \pm SD & Minimum & Maximum \\
\hline \multirow{2}{*}{$\begin{array}{l}\text { Age } \\
\text { (years) }\end{array}$} & Male & $48.17 \pm 20.13$ & 32 & 70 \\
\hline & Female & $55.58 \pm 18.95$ & 36 & 75 \\
\hline \multirow{4}{*}{$\begin{array}{l}\text { Vital } \\
\text { sign }\end{array}$} & Saturation (\%) & 95.98 & 85 & 100 \\
\hline & $\begin{array}{l}\text { Systolic blood } \\
\text { pressure }(\mathrm{mmHg})\end{array}$ & 151.58 & 85 & 220 \\
\hline & $\begin{array}{l}\text { Diastolic blood } \\
\text { pressure }(\mathrm{mmHg})\end{array}$ & 86.98 & 45 & 140 \\
\hline & Heart rate ${ }^{*}(/ \mathrm{dk})$ & 93.36 & 50 & 135 \\
\hline
\end{tabular}

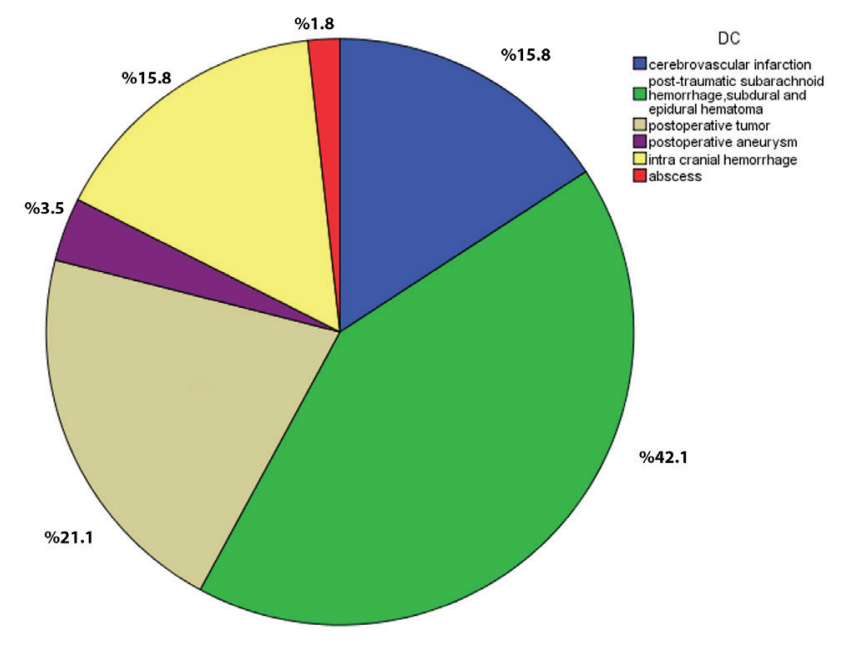

Graphic 1. Etiological diagnosis of patients who underwent decompressive craniectomy 
patient age grouping was made, when the mortality rates above and below the cut-off value of 40 years and 60 years were compared, it was statistically significant $(p<0.05)$. The death rate was found to be statistically significant due to the comparison of patients under the age of 40 with the patient group over 60 years old $(p<0.05)$.

The patients are classified as GCS. Patients with GCS between 3 and 7 were $35.1 \%(n=20), 36.8 \%(n=21)$ for GCS between 8 and 11, 28.1\% ( $n=16)$ for GCS between 12 and 15. Compared with the GCS group between 3 and 7 , the mortality is statistically significantly lower in GCS 12-15 ( $p<0.05)$ (Table 2).

The mean of midline shift in cranial CTs of all patients was $7.88 \pm 5.35 \mathrm{~mm}$. The average midline shift amount is $8.94 \pm 5.21 \mathrm{~mm}$ in the mortality group $(n=29)$ and was $6.79 \pm 5.23 \mathrm{~mm}$ in the survivor group $(n=28)$. Patients who did not develop shift were statistically significantly lower mortality $(p<0.05)$ than others who developed shifts, however, there was no statistically significant difference

\begin{tabular}{|c|c|c|c|c|c|c|}
\hline \multirow{5}{*}{$\begin{array}{l}\text { Midline } \\
\text { shift }\end{array}$} & \multirow{2}{*}{ GCS } & \multirow{2}{*}{ n } & \multirow{2}{*}{ Mean } & \multirow{2}{*}{ SD } & \multicolumn{2}{|c|}{$\begin{array}{l}95 \% \text { Confidence } \\
\text { interval }\end{array}$} \\
\hline & & & & & $\begin{array}{l}\text { Lower } \\
\text { bound }\end{array}$ & $\begin{array}{l}\text { Upper } \\
\text { bound }\end{array}$ \\
\hline & GCS: 3-7 & 20 & 8.67 & 4.77 & 37.62 & 108.87 \\
\hline & GCS: 8-11 & 21 & 7.87 & 5.19 & 78.26 & 143.54 \\
\hline & GCS: $12-15$ & 16 & 6.92 & 6.34 & 97.69 & 168.05 \\
\hline \multicolumn{5}{|l|}{ Overall } & 82.93 & 124.78 \\
\hline
\end{tabular}

\begin{tabular}{|c|c|c|c|c|c|c|c|c|}
\hline \multirow{2}{*}{\multicolumn{3}{|c|}{ Exitus }} & \multicolumn{6}{|c|}{ Midline shift } \\
\hline & & & No & $\begin{array}{l}\text { Less } \\
\text { than }\end{array}$ & From & From & $\begin{array}{l}\text { More } \\
\text { than }\end{array}$ & \\
\hline \multirow{4}{*}{ Yes } & \multirow{3}{*}{ Pupil } & Anisocoric & 0 & 2 & 3 & 2 & 3 & 10 \\
\hline & & $\begin{array}{l}\text { Fixdilate or } \\
\text { middilate }\end{array}$ & 0 & 0 & 4 & 1 & 2 & 7 \\
\hline & & Isochoric & 0 & 4 & 4 & 4 & 0 & 12 \\
\hline & \multicolumn{2}{|c|}{ Total } & 0 & 6 & 11 & 7 & 5 & 29 \\
\hline \multirow{4}{*}{ No } & \multirow{3}{*}{ Pupil } & Anisocoric & 1 & 1 & 5 & 4 & 1 & 12 \\
\hline & & $\begin{array}{l}\text { Fixdilate or } \\
\text { middilate }\end{array}$ & 0 & 0 & 0 & 0 & 0 & 0 \\
\hline & & Isochoric & 5 & 4 & 3 & 2 & 2 & 16 \\
\hline & \multicolumn{2}{|l|}{ Total } & 6 & 5 & 8 & 6 & 3 & 28 \\
\hline \multicolumn{3}{|c|}{ Total (n) } & 6 & 11 & 19 & 13 & 8 & 57 \\
\hline \multicolumn{9}{|c|}{ Independent t-test was used } \\
\hline
\end{tabular}

between groups that developed shifts in terms of mortality $(p>0.05)$.

The amount of shift was higher in elderly patients and it was statistically significant $(p<0.05)$. Under 40 years old $(5.55 \pm 4.40 \mathrm{~mm})$ midline shifts were found less and statistically significant compared to $40-60$ years old $(9.40 \pm 5.44 \mathrm{~mm})(p<0.05)$. When the shift rates under 40 years old $(5.55 \pm 4.40 \mathrm{~mm})$ and over 60 years old $(8.76 \pm 5.57 \mathrm{~mm})$ were compared, $p=0.05$ was found. Under 40 years of age $(5.55 \pm 4.40 \mathrm{~mm})$ midline shifts were less and statistically significant $(p<0.05)$ than over 40 years old $(9.05 \pm 5.45 \mathrm{~mm})$. Shift rates above 60 years old $(8.76 \pm 5.57)$ and below $(7.32 \pm 5.2)$ were not statistically significant $(p>0.05)$.

Statistical correlations were made between age, gender, preop and postop GCS of patients undergoing decompression surgery, surgical side, and presence of hypertension, development of midline shift, diabetes, anticoagulant use, duraplasty, and exitus. There was a statistical significance between anticoagulant use and GCS status, hypertension, and exitus of the group who underwent decompressive surgery $(p<0.05)$. In the case of anisocoric and fixed dilated pupils, the GCS of the patients were found to be low and statistically significant $(p<0.05)$.

As the patients age groups increase, the rate of using antihypertensive and anticoagulant use increases with a statistical correlation $(p<0.05)$. It was found that the patients with shift were statistically significantly higher between pupil status, all groups that went to decompressive surgery regarding mortality.

A statistical significance was found between fixed and mid-dilated pupils and exitus $(p<0.05)$. All patients with fixed or mid-dilated patients $(n=7)$, twelve patients $(42.85 \%)$ with pupillary isochoric and light reflexes, ten (45.45\%) of the patients with anisocoria have died.

The midline shifts of the patients with anisocoria were $8.64 \pm 5.00 \mathrm{~mm}$, and in the patients with fix dilated or mid-dilated were $10.27 \pm 5.98 \mathrm{~mm}$. Patients with isochoric pupils have a midline shift of $6.70 \pm 5.34 \mathrm{~mm}$. A statistical significance was found between fixed or mid-dilated in terms of mortality $(p<0.05)$. No statistical significance was found when the patients with and without anisocoria in terms of mortality $(p>0.05)$. Pupil states of the patients were compared with each shift group. There was no statistical significance ( $p>0.05$ ) (Table 3 ).

\section{Discussion}

DC has been increasingly applied recently (9). DC is frequently applied to MCA infarcts. The rate of infarction cases is 10-20 per hundred thousand per year. Mortality rates range from $41 \%$ to $79 \%$, according to literature. While the mortality rate is $16 \%$ in patients with early 
surgery, it reaches $34.4 \%$ in the late period and $78 \%$ in the non-surgical group $(10,11)$. In addition, it has been stated that right hemisphere involvement, advanced age, and presence of pupil dilation will increase the mortality rate in MCA infarct patients who have been applied DC (12). The average age of our patient group with cerebral infarction was 64.55. DC was applied after the patients had neurological and clinical deterioration who did not improve despite medical treatments. The mortality rate was $55.5 \%$.

DC can be applied early or late. Early DC is usually performed in the acute phase, and hemorrhage is intervened to reduce intracranial pressure. Late DC is applied to patients who do not benefit from medical treatment, who have increased intracranial pressure or do not decrease pressure levels (13). Studies show that DC has a better prognosis than medical treatment and is more effective in controlling ICP levels (1). It has been shown that late DC has a lower prognosis effect than early DC (14). Early DC and hemorrhage evacuation were performed in patients with intracranial hemorrhage after head trauma. The mortality rate of trauma patients was $58.3 \%$. In recent studies, it has been stated that despite the decrease in acute DC applications to trauma patients, there may be a decrease in mortality rates with medical treatment and good care (15). Compared to infarct patients, the mean age of trauma patients was low, had a lower midline shift, and the mortality rate was higher despite early DC application. This result may be due to brain damage, cerebral edema, and the extent of brain ischemia due to decreased perfusion pressure. Pupils of six of the trauma patient group were fixed and mid-dilated. The pupils of six of the trauma patient group were fixed and moderately dilated. The amount of midline deviation was significantly higher in these patients compared to the other patient groups. Early DC was applied, and all six patients died. Pupillary was fix-dilated, and the exitus rate was high due to the mass and edema effect and the excess amount of shift. In the literature, it has been reported that patients with intracerebral hemorrhage have a poor prognosis if their mean GCS is $<8$ and the average age is $>58.18 \pm 14.22$, and the amount of shift is $>4 \mathrm{~mm}(16)$. However, it was stated that the mortality rate of patients with GCS $<5$ and bilaterally fixed dilated pupils would be extremely high (17). In our patient group with intracerebral hemorrhage, the mean GCS is 8.2, and the average age is $49.77 \pm 17.59$. The shift was $9.08 \pm 6.27$. Five patients died (55.5\%). Despite the early application of DC to these patients, GCS showed a poor prognosis due to low and high midline shifts. Bleeding occurred in 12 patients after intracranial tumor surgery; early DC applied. The mortality rate was $33.3 \%$. After aneurysm surgery, bleeding developed in two patients and one patient died. The mortality rate was $50 \%$. The mortality rate was found to be lower than bleeding after intracranial tumor surgery and bleeding after aneurysm surgery. Due to the low number of patients with hematoma after aneurysm surgery, the mortality rate may have been high. One patient was operated on for intracranial abscess, and DC was applied due to hemorrhage development, and the patient recovered completely. Mortality rates of secondary intracranial hemorrhages were found to be lower than primary intracranial hemorrhages.

In the patient groups, the amount of shift was lower in those under 40 years of age compared to other age groups ( $5.55 \pm 4.40 \mathrm{~mm}$ versus $9.05 \pm 5.45 \mathrm{~mm}$ ). Because young patients have good brain compliance and elastance, they are resistant to lesions, and their success rate increases with faster diagnosis and early application of DC because they show clinical signs in fewer shift conditions. In elderly patients, the brain tissue's compliance and elastance are low and show less resistance to lesions. When the amount of shift reaches advanced levels, clinical findings emerge. Therefore, it causes late diagnosis and initiation of late treatment. In our study, exitus did not develop in patients who did not develop midline shift. Edema in the brain tissue causes an increase in intracranial pressure, decreased perfusion pressure, and ischemia. When DC is performed, intracranial pressure decreases with brain tissue expansion, and cerebral perfusion improves. Although DC effectively controls intracranial pressure, the administration time to patients is controversial $(18,19)$. In our study, we evaluated pupil diameters and DC application times and results. The mean midline level of the group with pupil status, fix dilated or mid-dilated, is $10.27 \pm 5.98 \mathrm{~mm}$. Early-term DC was applied to the patients in this group. All patients with fixed or mid dilated pupils have died.

The absence of light reflex indicates a poor prognosis. Twelve $(42.85 \%)$ of the patients with pupillary isochoric and bilateral light reflexes and 10 (45.45\%) of the patients with anisocoria died. Midline shifts are higher in anisocoric patients. It will be insufficient to decide on DC time by evaluating pupil diameters alone. Rapid and early application of DC in anisocoric pupils may affect decreasing mortality. Besides, low GCS, high age, and comorbid diseases were factors affecting mortality. In one study, patients with brain damage causing increased intracranial pressure were evaluated by comparing those who received DC and medical treatment. Patients' mortality after decompression surgery was found to be lower than those who received conservative treatment. However, he had high vegetative survival and bedriddenness $(20,21)$. In our study, mortality rates of patients with isochoric pupils 
treated with DC were found to be lower than those with middilate pupils.

\section{Study Limitations}

The low number of patients caused us to make limited evaluations. A larger number of patients are needed for a more comprehensive assessment. There is heterogeneity between intracranial pathologies. Therefore, the timing of the operations may have caused differences in the preoperative and postoperative neurological examination results. Despite these limitations, our study will contribute to the literature in terms of providing an idea about mortality and decompressive surgery time by evaluating pupil diameter and midline shift conditions.

\section{Conclusion}

The mortality of patients after decompression surgery is lower than those who received conservative treatment. However, it has high vegetative survival and bed dependency. As the time of DC, it will be beneficial to decrease mortality if it is preferred in the early period without waiting for asymmetry in pupil diameters or loss of light reflex.

\section{Authorship Contributions}

Concept: A.A., Design: U.E., G.B., Data Collection or Processing: A.A., Analysis or Interpretation: U.E., Literature Search: G.B., Writing: G.B., A.A.

Conflict of Interest: No conflict of interest was declared by the authors.

Financial Disclosure: The authors declared that this study received no financial support.

\section{References}

1. Hutchinson PJ, Kolias AG, Timofeev IS, et al. Trial of Decompressive Craniectomy for Traumatic Intracranial Hypertension. N Engl J Med 2016;22:1119-30.

2. Badri S, Chen J, Barber J, et al. Mortality and long-term functional outcome associated with intracranial pressure after traumatic brain injury. Intensive Care Med 2012;38:1800-9.

3. Schur S, Martel P, Marcoux J. Optimal bone flap size for decompressive craniectomy for refractory increased intracranial pressure in traumatic brain injury: taking the patient's head size into account. World Neurosurg 2020;137:430-6.

4. Panourias IG, Skiadas PK, Sakas DE, Marketos SG. Hippocrates: A Pioneer in the Treatment of Head Injuries. Neurosurgery 2005;57:181-9.

5. Brown DA, Wijdicks EFM. Decompressive craniectomy in acute brain injury. Handb Clin Neurol 2017;140:299-318.

6. Timofeev I, Hutchinson PJ. Outcome after surgical decompression of severe traumatic brain injury. Injury 2006;37:1125-32.
7. Qiu W, Guo C, Shen H, et al. Effects of unilateral decompressive craniectomy on patients with unilateral acute post-traumatic brain swelling after severe traumatic brain injury. Crit Care 2009;13:185.

8. Cooper DJ, Rosenfeld JV, Murray L, et al. Decompressive Craniectomy in Diffuse Traumatic Brain Injury. N Engl J Med 2011;364:1493-502.

9. Timofeev I, Santarius T, Kolias AG, Hutchinson PJA. Decompressive craniectomy - operative technique and perioperative care. Adv Tech Stand Neurosurg 2012;38:115-36

10. Elsawaf A, Galhom A. Decompressive Craniotomy for Malignant Middle Cerebral Artery Infarction: Optimal Timing and Literature Review. World Neurosurgery 2018;116:71-8.

11. Tagliaferri F, Zani G, laccarino C, et al. Decompressive craniectomies, facts and fiction: a retrospective analysis of 526 cases. Acta Neurochir (Wien) 2012;154:919-26.

12. Chen X, Hao Q, Yang SZ, et al. Improvement in Midline Shift Is a Positive Prognostic Predictor for Malignant Middle Cerebral Artery Infarction Patients Undergoing Decompressive Craniectomy. Front Neurol 2021;12:652827.

13. Nirula R, Millar D, Greene T, et al. Decompressive craniectomy or medical management for refractory intracranial hypertension: An AAST-MIT propensity score analysis. J Trauma Acute Care Surger 2014;76:944-55.

14. Wang R, Li M, Gao WW, Guo Y, Chen J, Tian HL. Outcomes of Early Decompressive Craniectomy Versus Conventional Medical Management After Severe Traumatic Brain Injury: A Systematic Review and Meta-Analysis. Medicine (Baltimore) 2015;94:1733.

15. Posti JP, Luoto TM, Rautava P, Kytö V. Mortality After Trauma Craniotomy Is Decreasing in Older Adults-A Nationwide Population-Based Study. World Neurosurg 2021;152:313-20.

16. Yang WS, Li Q, Li R, et al. Defining the Optimal Midline Shift Threshold to Predict Poor Outcome in Patients with Supratentorial Spontaneous Intracerebral Hemorrhage. Neurocrit Care 2018;28:314-21.

17. Tang Z, Yang R, Zhang J, et al. Outcomes of Traumatic BrainInjured Patients With Glasgow Coma Scale $<5$ and Bilateral Dilated Pupils Undergoing Decompressive Craniectomy. Front Neurol 2021;12:656369.

18. Kolias AG, Kirkpatrick PJ, Hutchinson PJ. Decompressive craniectomy: past, present and future. Nat Rev Neurol 2013;9:405-15.

19. Bohman LE, Schuster JM. Decompressive Craniectomy for Management of Traumatic Brain Injury: An Update. Curr Neurol Neurosci Rep 2013;13:392.

20. Lu G, Zhu L, Wang X, Zhang H, Li Y. Decompressive Craniectomy for Patients with Traumatic Brain Injury: A Pooled Analysis of Randomized Controlled Trials. World Neurosurg 2020;1:135-48.

21. Lin J, Frontera JA. Decompressive Hemicraniectomy for Large Hemispheric Strokes. Stroke. 2021;52:1500-10. 